\title{
Características de los consumidores de drogas recreativas en España y otros países europeos
}

\author{
Cesáreo Fernández Gómez
}

Irefrea - España

Enviar correspondencia: Cesáreo Fernández Gómez. B Samese, 52 A. 48114 ARRIETA (VIZCAYA) cesareo@cidecot.org

\section{Resumen}

Introducción: Los modelos predictivos del uso de drogas incluyen la personalidad, características familiares y de los pares y variables mediadoras (expectativas, motivación, percepción de riesgos, etc.). El estudio pretende valorar la capacidad predictiva relativa de los estilos recreativos junto con los predictores anteriores para distinguir el uso o no uso de drogas.

Material y método: Dos muestras de jóvenes, balaceadas entre consumidores y no - consumidores de drogas, una española $(N=806)$ y otra procedente de 10 ciudades europeas $(N=1777)$. Se utilizaron análisis discriminantes y de regresión logística para clasificar a los sujetos consumidores y no consumidores, estimar la capacidad predictiva relativa de cada grupo de variables y comparar los resultados en las dos muestras.

Resultados: El análisis discriminante clasificó correctamente alrededor del $90 \%$ de los entrevistados como consumidores o no consumidores. La personalidad, características de los pares y familiares y las variables mediadoras fueron incluidas en el modelo, aunque la gestión del ocio del fin de semana resultó el mejor predictor.

Conclusión: La prevención del uso de drogas debería incluir la gestión de la vida recreativa como un factor relevante, junto con el uso de drogas entre los padres y familiares, la personalidad y variables mediadoras.

Palabras claves: uso de drogas, pares, familia, estilos recreativos, personalidad.

\section{Summary}

Background: Predictive models on substance use include personality, peers and family characteristics and mediating variables (expectancies, motivation, risk perception, etc.). This study aim to compare the relative capacity of management of recreational life with the mentioned predictors, when the objective is to "predict" substance use.

Method: Two samples of young people were interviewed, both balanced between substance users and non - users. One were composed only by Spanish people $(\mathrm{N}=806)$ and the other was composed by people from ten European cities ( $N=1,777)$. Discriminant analyses and logistic regression methods were used to classify individuals regarding their status (user and non - user), to estimate the relative weight of different domains of variables in the models and to compare findings in the two samples.

Results: Similar findings were obtained across the two samples. A discriminant model classified around $90 \%$ of individuals correctly as users and non - users. Personality, peers and family characteristics and mediating variables were included. Management of recreational life was the stronger predictor, followed by peers substance use.

Conclusion: Substance use prevention should include management of recreational life as a relevant factor, together with another determinants as peers and family substance use, personality and mediating variables.

Key Words: Drug-use, going-out, peers, family, personality. 


\section{MARCOY ENCUADRE}

os estudios conducidos recientemente por Irefrea desde el Proyecto SONAR (Calafat et al., 1998, 1999, 2000, 2001) han aportado evidencia acumulativa a favor de un modelo sobre el desarrollo del uso y abuso de drogas entre los jóvenes españoles y de otros países europeos. Este modelo considera el uso y abuso recreativo de drogas como un patrón conductual multideterminado, y se aproxima a su estudio desde una perspectiva tridimensional que integra variables relativas a las drogas, el sujeto y su entorno. Nuestros estudios han hallado evidencia acumulativa de la asociación entre el uso de drogas y determinadas características de la personalidad del sujeto (una predisposición hacia las conductas de riesgo, la búsqueda de sensaciones y la desviación social). De modo similar a los hallazgos de otros estudios, el uso de drogas en los estudios de Irefrea también está asociado positivamente a características del entorno grupal y familiar del sujeto tales como el consumo de drogas, menor integración y control familiar, menor orientación hacia actividades pro-sociales, etc. Los factores que emergen como más relevantes en la interacción sujeto - sustancia en estos estudios parecen ser el valor funcional e instrumental del consumo en la integración social de determinados sujetos, y, muy especialmente, su valor funcional e instrumental como "nexo" alrededor del cual gira la vida recreativa del fin de semana en determinados entornos culturales, "tribus" y grupos de adolescentes y jóvenes. Este valor funcional del consumo de drogas en algunos jóvenes está caracterizado por una serie de expectativas acerca de los efectos de diversas sustancias, una percepción de la "utilidad" de consumir drogas y motivaciones en pro y en contra de este consumo, de una determinada percepción acerca de la presencia o ausencia de riesgos asociados al consumo, y de determinadas actitudes hacia el consumo de drogas en la red social de referencia y en la comunidad.
El componente original de este marco de referencia de Irefrea es que combina el estudio de estos factores etiológicos del consumo de drogas (personales, del entorno social - familiar y motivacionales - cognitivos) con el estilo de gestión de la vida recreativa durante el fin de semana.

El objetivo de este capítulo es proporcionar una visión global de los principales factores asociados al uso o no uso de drogas entre los jóvenes entrevistados por Irefrea en entornos recreativos en España durante el año 1999 y en 10 países europeos durante el año 2001. La hipótesis principal es que el uso de drogas entre muchos jóvenes europeos se asocia a un estilo de vida orientado hacia el riesgo y la búsqueda de sensaciones, y que gestiona la diversión añadiéndole a su significado principal y genuino el uso de drogas como una "herramienta". Los espacios recreativos del fin de semana proporcionan un espacio donde estos jóvenes consumidores desarroIlarían un estilo de diversión diferente al de otros jóvenes que también dedican un tiempo sustancial del fin de semana a la diversión, sin incluir el consumo de drogas como actividad lúdica y que mantienen mayor orientación hacia otras actividades cotidianas junto con una actitud que no justifica ni apoya el consumo de drogas en sus grupos de referencia y en la comunidad.

Los objetivos específicos que se persiguen son los siguientes:

1. Identificar factores relevantes en su asociación con el uso o no uso de drogas. Estos factores se agrupan en diferentes áreas o conjuntos de variables: entorno familiar - social, factores motivacionales cognitivos asociados al uso de drogas, características relacionadas con la personalidad y estilos de gestión de la vida recreativa durante el fin de semana.

2. Construir un modelo predictivo, combinando las variables más asociadas al uso de drogas y valorar la capacidad predictiva relativa de los mencionados dominios o grupos de variables. 
3. Valorar la capacidad del modelo para predecir el uso de drogas.

\section{MATERIAL Y MÉTODO}

\section{Muestras}

Las muestras descritas en este capítulo están formadas por jóvenes entrevistados por Irefrea en España durante el Otoño del año 1999 y en otros países europeos entre Marzo y Julio del 2001. Se han elegido muestras de jóvenes procedentes de cuatro ciudades españolas en el año 1999 y de diez ciudades europeas en 2001. Todos ellos son jóvenes que salen con frecuencia a divertirse los fines de semana en clubs, pubs, discos, etc. La mitad de la muestra son no consumidores o consumidores muy moderados y la otra mitad cosumidores de alcohol y otras drogas. Como consecuencia del diseño del estudio, las muestras están relativamente balanceadas según tres variables de agrupación: género, consumo / no consumo de drogas y grupo de edad. El cuadro siguiente describe los criterios utilizados para definir el estatus de los encuestados como consumidores o no-consumidores:

\section{CONSUMIDORES Y NO-CONSUMIDORES}

Para los fines de estos estudios, un consumidor es una persona que, además de consumir habitualmente alcohol y / o tabaco, también consume alguna droga ilegal.

Un no-consumidor es una persona que:

- No consume drogas ilegales

- No ha consumido tabaco más de tres veces durante el mes anterior

- Si ha fumado en ese mes, no más de tres cigarrillos al día

- No se ha emborrachado durante el ultimo año ni ha tomado alcohol más de cuatro días durante el mes anterior

- Si ha tomado alcohol durante ese mes, no más de dos bebidas en una misma sesión

No-Consumidores pueden ser ex-consumidores o haber llegado solamente a probar alguna sustancia ilegal.
La tabla $n^{0} 1$ describe la composición de las muestras y su segmentación según género, grupos de edad y estatus en relación al consumo de drogas. Los denominados "adolescentes" son sujetos con edad máxima de 18 años, y los denominados "jóvenes" son sujetos de edades superiores a los 19 años. La media de edad en ambas muestras (española y europea) es algo superior a los 20 años.

La tabla $\mathrm{n}^{\circ} 2$ muestra las frecuencias y porcentajes válidos relativos a los diferentes lugares de residencia en ambas muestras. Algo más de la mitad de los sujetos de la muestra europea viven con la familia de origen $(59,5 \%)$, mientras que en la muestra española son una gran mayoría $(84,7 \%)$. Alrededor de uno de cada 10 viven solos, en pareja o con amigos y alrededor del 10\% restante vive en una residencia o en otro lugar en la muestra europea, mientras que esos porcentajes son menores en la muestrea española.

Dos de cada tres entrevistados o más estudia como actividad principal. Uno de cada cinco trabaja regularmente en la muestra europea, aunque ese porcentaje en la muestra española apenas supera el $10 \%$. Un pequeño porcentaje (entre el 3 y $7 \%$ ) trabajan de modo temporal, están desempleados o están en otra situación ocupacional.

La mitad de los sujetos encuestados proceden de familias con estatus socioeconómico medio, uno de cada tres proceden de familias con nivel socioeconómico medio alto o alto y alrededor del $15 \%$ proceden de familias con un estatus medio - bajo o bajo.

Las muestras están relativamente balanceadas por ciudades. En el estudio europeo algunas de ellas (Utrecht, Liverpool, Lisboa y Turku) están representadas con un menor número de sujetos encuestados. Los sujetos entrevistados en Palma son los mismos en ambos estudios.

La base principal de los resultados de este capítulo se centra en comparar a los sujetos consumidores y no consumidores entrevistados por Irefrea en ambos estudios, realizados en el año 1999 y el año 2001. Como se ha mencionado anteriormente, los sujetos con- 


\begin{tabular}{|c|c|c|c|}
\hline \multirow[b]{2}{*}{ Variable } & \multicolumn{3}{|c|}{$\begin{array}{l}\text { Tabla } n^{\circ} 1 \text { : Composición y segmentación por sexo, grupo de edad } \\
\text { y consumo de drogas en las dos muestras (Española y Europea). }\end{array}$} \\
\hline & $\begin{array}{l}\text { categoría } \\
\text { / estadístico }\end{array}$ & $\begin{array}{l}1999(\mathrm{n}=806) \\
\text { Española }\end{array}$ & $\begin{array}{l}2001(\mathrm{~N}=1777) \\
\text { europea }\end{array}$ \\
\hline \multirow[t]{2}{*}{ SEXO } & Hombres & $407(50,5 \%)$ & $861(48,5 \%)$ \\
\hline & Mujeres & $399(49,5 \%)$ & $916(51,5 \%)$ \\
\hline \multirow[t]{2}{*}{ CONSUMO } & Consumidores & $397(49,3 \%)$ & $943(53 \%)$ \\
\hline & No-Consumidores & $409(50,7 \%$ & $834 / 47 \%)$ \\
\hline \multirow[t]{2}{*}{ GRUPO DE EDAD } & Adolescentes & $401(49,8 \%)$ & $878(49,4 \%)$ \\
\hline & Jóvenes & $405(50,2 \%)$ & $899(50,6 \%)$ \\
\hline \multirow[t]{3}{*}{ EDAD } & Media & 20,2 & 20,3 \\
\hline & Desviación Típica & 4,2 & 3,8 \\
\hline & Rango & $21(13-34)$ & $23(13-36)$ \\
\hline
\end{tabular}

\begin{tabular}{|lcc|}
\hline \multicolumn{3}{|c|}{$\begin{array}{c}\text { Tabla no 2: } \\
\text { 2ujetos de las dos muestras } \\
\text { sujes donde viven los }\end{array}$} \\
Convivencia & España, 1999 & Global europa, 2001 \\
\hline FAMILIA & $683(84,7 \%)$ & $1041(59,5 \%)$ \\
\hline PAREJA & $46(5,7 \%)$ & $176(10,1 \%)$ \\
\hline AMIGOS & $35(4,3 \%)$ & $151(8,6 \%)$ \\
\hline SOLO / A & $21(2,6 \%)$ & $214(12,2 \%)$ \\
\hline RESIDENCIA & $5(0,6 \%)$ & $124(7,1 \%)$ \\
\hline OTROS & $16(2 \%)$ & $43(2,5 \%)$ \\
\hline
\end{tabular}

sumidores son personas que se declaran al menos consumidores de alguna droga ilegal, además de ser casi todos consumidores habituales de drogas legales. El gráfico 1 muestra los porcentajes de sujetos consumidores que declaran consumir diferentes sustancias legales e ilegales.

La gran mayoría de los entrevistados etiquetados como consumidores lo eran de alcohol, tabaco y cannabis. El consumo de otras drogas ilegales era más frecuente en la muestra europea que en la española.

Además, la gran mayoría de estos consumidores admiten que se han embriagado al menos una vez durante el mes anterior (76,5\% en el estudio español y 73,3\% en el estudio europeo) y más de la mitad en dos o más ocasiones durante el mismo mes anterior $(52 \%$ en el estudio español y $53 \%$ en el europeo). Así pues, cuando a lo largo de este
Tabla $n^{\circ}$ 3: Ocupación en las muestras entrevistadas

\begin{tabular}{lcc|} 
OCUPACIÓN & España, 1999 & Global europa, 2001 \\
\hline ESTUDIANTE & $588(73 \%)$ & $1069(65,2 \%)$ \\
\hline TRABAJO TEMPORAL & $56(7 \%)$ & $110(6,7 \%)$ \\
\hline TRABAJO PERMANENTE & $91(11,3 \%)$ & $326(19,9 \%)$ \\
\hline DESEMPLEADO & $47(5,8 \%)$ & $61(3,7 \%)$ \\
\hline OTRA SITUACIÓN & $24(3 \%)$ & $73(4,5 \%)$ \\
& & \\
\hline
\end{tabular}

capítulo nos referimos a los "sujetos consumidores" estamos hablando de dos subgrupos formados por sujetos que consumen casi en su totalidad alcohol, tabaco y cannabis, que consumen otras drogas ilegales en porcentajes comprendidos entre el 8 y $44 \%$ y que en su mayoría consumen alcohol en exceso.

\section{Procedimientos}

Obtención de datos

El instrumento utilizado para la obtención de datos en ambos estudios es una entrevista estructurada con cuestiones relativas a: 1) características sociodemográficas, 2) rasgos personales: desviación social, conductas de riesgo y búsqueda de sensaciones; 3) religión e ideología política; 4) estilos de gestión de la vida recreativa del fin de semana; 5) variables 
Tabla $n^{\circ}$ 4: Nivel socioeconómico familiar

\begin{tabular}{|lcc|} 
nivel socioeconómico & España, 1999 & Global Europa, 2001 \\
\hline ALTO & $39(4,9 \%)$ & $119(6,8)$ \\
\hline MEDIO - ALTO & $236(29,4 \%)$ & $489(27,8)$ \\
\hline MEDIO & $429(53,4 \%)$ & $887(50,4)$ \\
\hline MEDIO - BAJO & $85(10,6 \%)$ & $207(11,8)$ \\
\hline BAJO & $14(1,7 \%)$ & $58(3,3)$ \\
\hline
\end{tabular}

Tabla ${ }^{\circ}$ 5: Ciudad de procedencia de los sujetos encuestados

\begin{tabular}{|lcc|}
\hline CIUDAD & Frecuencia & Porcentaje \\
\hline LISBOA & 167 & 9,4 (año 2001) \\
\hline NIZA & 197 & 11,1 (año 2001) \\
\hline BOLONIA & 198 & 11,1 (año 2001) \\
\hline VIENA & 179 & 10,1 (año 2001) \\
\hline LIVERPOOL & 157 & 8,8 (año 2001) \\
\hline BERLIN & 195 & 11 (año 2001) \\
\hline TURKU & 161 & 9,1 (año 2001) \\
\hline ATENAS & 202 & 11,4 (año 2001) \\
\hline UTRECHT & 121 & 6,8 (año 2001) \\
\hline PALMA & 205 & $24,7 \%$ (1999) y 11,3 (año 2001) \\
\hline MADRID & 199 & $24,9 \%$ (año 1999) \\
\hline VIGO & 201 & $24,9 \%$ (año 1999) \\
\hline BILBAO & 201 & \\
\hline
\end{tabular}

Gráfico 1: Porcentajes de consumidores de diferentes sustancias, entre el subgrupo de "consumidores".

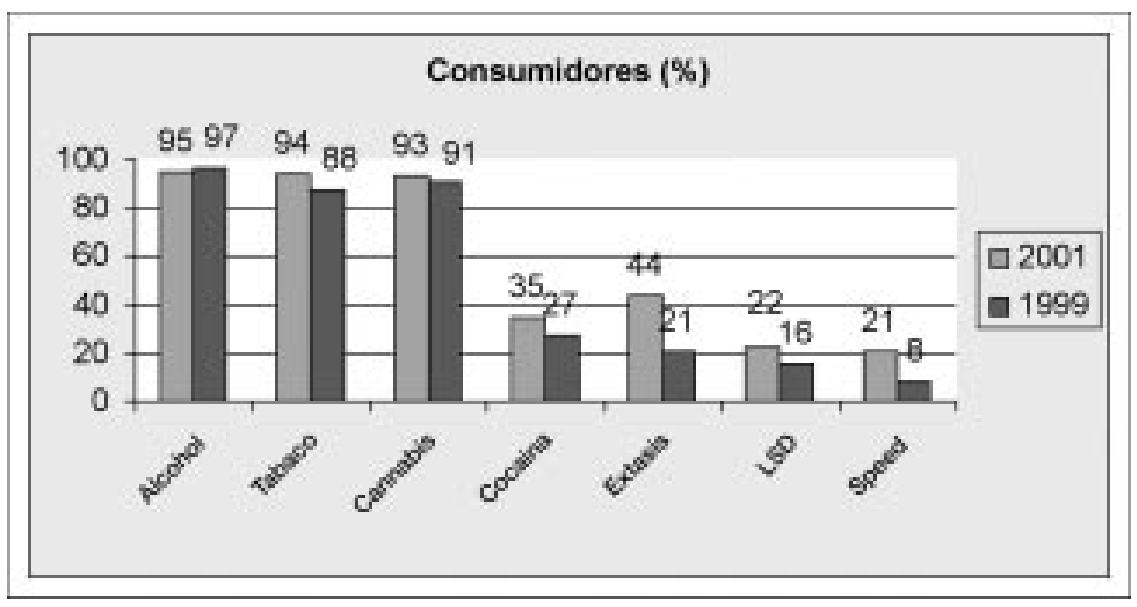


del entorno social y familiar; 6) actitudes, percepción de riesgos y expectativas relacionadas con el consumo de drogas. El contenido de estas secciones de la encuesta será descrito más adelante, junto con los resultados obtenidos en cada sección.

\section{Análisis de los datos}

Para alcanzar los objetivos específicos marcados anteriormente, hemos utilizado el análisis discriminante y la regresión logística, según que las variables que utilizamos para distinguir a los sujetos que consumen o no consumen drogas sean cuantitativas o cualitativas respectivamente. Cuando utilizamos la regresión logística, presentamos los coeficientes de cada variable en la ecuación de regresión logística y los estadísticos alcanzados por el modelo a la hora de "clasificar" a cada sujeto según su grupo de pertenencia (consumidor o no consumidor de drogas). La interpretación de la ecuación de regresión logística es relativamente simple, ya que el valor de los coeficientes de las variables es un indicador del incremento en la probabilidad de ser consumidor drogas cuando la variable cambia de valor. Los coeficientes positivos indican un aumento de la probabilidad, mientras que los negativos implican una disminución. En los modelos basados en el análisis multivariante, los resultados incluyen los coeficientes de cada variable en una función discriminante que distingue consumidores y no consumidores, indicando el peso que dicha variable tiene en la función resultante. Además, se exponen los resultados alcanzados por el modelo en la clasificación de los sujetos en un grupo de "consumidores" o "no consumidores" de drogas.

La "probabilidad a priori" de pertenecer al grupo de consumidor o no consumidor es muy similar, de modo que la capacidad de cada modelo para clasificar a cada sujeto en su grupo es atribuible a la capacidad discriminativa de las variables que lo forman, más que al hecho de que ambas probabilidades sean muy distintas. Las variables "predictoras", "independientes" o "covariables" son introducidas en el modelo siguiendo el procedimiento "paso a paso". En la regresión logística utilizamos el modelo denominado "adelante: condicional" para la inclusión de variables. En el análisis discriminante se utilizó el método de inclusión por pasos de las variables "predictoras", basado en la distancia de Mahalanobis. Solamente son comentadas y discutidas las variables que son incluidas en los modelos con un "peso relativo" estadísticamente significativo. Asimismo, todos los modelos comentados y discutidos más adelante alcanzan estadísticos de ajuste adecuados y niveles de significación apropiados para ser interpretados sustantivamente.

\section{CARACTERÍSTICAS PERSONALES}

En este apartado se describen las asociaciones entre algunas características psicológicas y conductuales del sujeto y el consumo / no consumo de drogas. En estos dos estudios de encuestas se han incluido, además de las características sociodemográficas básicas, 4 constructos relativos a las características personales del sujeto:

1. Desviación social,

2. Conductas de riesgo relacionadas con la conducción de vehículos,

3. Predisposición hacia la búsqueda de sensaciones, y

4. Creencias religiosas e ideología política.

\subsection{Desviación social y consumo de drogas}

Los indicadores de desviación social utilizados en los dos estudios han sido los siguientes, todos ellos evaluados de modo dicotómico en respuesta a si el sujeto los ha realizado alguna vez en el ciclo vital (si / no):

1. Conducir vehículos sin licencia,

2. Vandalismo (dañar o romper cosas en lugares públicos),

3. Hurtos (llevarse cosas sin pagar de lugares comerciales), y 
4. Violencia (pelearse con personas que no son de la familia).

Estas cuatro conductas "desviadas" socialmente han sido realizadas alguna vez en su vida por porcentajes que varían entre el $28 \%$ (vandalismo) y el 61,2\% (la participación en peleas), siendo más frecuentes todas ellas entre los consumidores que entre los no-consumidores. La tabla $n^{\circ} 6$ resume los resultados obtenidos con un modelo de regresión logística aplicado a estas conductas indicadoras de desviación social y conducta delictiva.

El signo positivo de los tres coeficientes indica que la probabilidad de ser consumidor de drogas aumenta si el sujeto ha realizado en su vida alguna de las tres conductas desviadas. La asociación entre las dos conductas excluidas y consumo de drogas está explicada por las variables incluidas en las dos ecuaciones de regresión logística.

La magnitud de los coeficientes muestra que la participación en hurtos es claramente la que mejor "predice" el consumo de drogas, dentro de las tres conductas desviadas. Globalmente, el modelo viene a decir que la historia del sujeto, relativa solamente a estas tres conductas antisociales y desviadas es capaz de "predecir" alrededor de dos tercios de la conducta de consumir o no consumir drogas en las dos muestras estudiadas.

\subsection{Conductas de riesgo y consumo de drogas}

Los indicadores de conductas de riesgo han sido los siguientes, todos ellos relativos a la conducción de vehículos bajo los efecto de alcohol y drogas, y evaluados de modo dicotómico en respuesta a si el sujeto los ha realizado alguna vez durante el ciclo vital (si / no) o si estaría dispuesto a realizarlos:

1. Conducir vehículos bajo los efectos del alcohol,

2. Viajar en un vehículo conducido por alguien bebido,

3. Viajaría en un vehículo conducido por alguien bajo los efectos de otras drogas, y

4. Si o no evitaría que un amigo condujese bebido.

La tabla $n^{0} 7$ resume los resultados obtenidos con un modelo de regresión logística aplicado a estas conductas de riesgo en la conducción de vehículos.

Nuevamente observamos resultados muy similares en los estudios español y europeo: El factor más discriminativo en ambos estudios es la actitud favorable a viajar con un conductor bajo los efectos de las drogas, seguido de la probabilidad de haber conducido estando bebido.

Los coeficientes positivos en las variables predictoras significan que la probabilidad de

\begin{tabular}{|lcc|}
\hline \multicolumn{3}{|c|}{$\begin{array}{c}\text { Tabla } \mathbf{n}^{\mathbf{0}} \text { 6: Desviación social y predicción del } \\
\text { consumo o no consumo de drogas } \\
\text { CARACTERÍSTICAS PERSONALES }\end{array}$} \\
\hline \multicolumn{3}{|c|}{ Desviación social } \\
\hline Variable Estudio español & Estudio europeo \\
\hline Hurtos & 1,1 & 1 \\
\hline Vandalismo & $n .5$. & 0,46 \\
\hline Conducir vehículos sin licencia & 0,56 & 0,29 \\
\hline Participación en peleas & 0,79 & $n . s$. \\
\hline Constante & $-1,1$ & $-2,96$ \\
\hline $\begin{array}{l}\text { Capacidad clasificatoria del modelo } \\
\text { que incluye los cuatro parámetros }\end{array}$ & $68 \%$ & $65 \%$ \\
\hline
\end{tabular}




\begin{tabular}{|c|c|c|}
\hline \multicolumn{3}{|c|}{$\begin{array}{l}\text { Tabla } n^{\circ} \text { 7: Conductas de riesgo en la conducción de } \\
\text { vehículos y consumo o no consumo de drogas. }\end{array}$} \\
\hline \multicolumn{3}{|c|}{ CARACTERÍSTICAS PERSONALES } \\
\hline \multicolumn{3}{|c|}{ Conductas de riesgo } \\
\hline Variable & Estudio español & Estudio europeo \\
\hline Ha conducido bebido & 1,4 & 1,1 \\
\hline Ha viajado con alguien bebido al volante & 1,1 & 0,8 \\
\hline Viajaría con alguien drogado al volante & 1,6 & 1,2 \\
\hline Evitaría que un amigo condujese bebido & n.s. & n.s. \\
\hline Constante & $-6,9$ & $-5,3$ \\
\hline $\begin{array}{l}\text { Capacidad clasificatoria del modelo } \\
\text { que incluye los cuatro parámetros }\end{array}$ & $73,2 \%$ & $70,3 \%$ \\
\hline
\end{tabular}

estas conductas de riesgo son mayores entre los consumidores de drogas que entre los no-consumidores. Se puede concluir que la historia de haber realizado alguna vez o no estas conductas o estar dispuesto a realizarlas predice por si sola más de $2 / 3$ del hecho de ser o no consumidor de drogas en nuestras dos muestras.

\subsection{Búsqueda de sensaciones y consumo de drogas}

Se han utilizado tres indicadores de búsqueda de sensaciones, todos ellos evaluados con una escala de 6 puntos ordenados de menor a mayor frecuencia durante el ciclo vital y el último año:

1. Hacer lo que apetece, sea lo que fuere,

2. Hacer algo peligroso porque alguien le desafió a ello, y

3. Hacer cosas "locas", aunque sean un poco peligrosas.

La tabla n8 resume los resultados del análisis discriminante aplicado a estos indicadores. Nuevamente hallamos resultados casi totalmente coincidentes en ambos estudios:

1. Dos de estos indicadores de búsqueda de sensaciones han sido incluidos en el modelo, y el más relevante es la frecuencia de hacer cosas "locas", aunque sean peligrosas.
2. La función canónica discriminante basada en estos indicadores alcanza una capacidad moderada para clasificar correctamente a los sujetos según u consumo / no consumo de drogas: algo menos de dos de cada tres sujetos con clasificados correctamente.

Los dos ítems incluidos indican comportamientos significativamente más frecuentes entre los consumidores que entre los no-consumidores.

\subsection{Religión, ideología y consumo de dro- gas}

Las creencias religiosas y la ideología política fueron evaluadas con una escala de 5 puntos, donde las máximas puntuaciones corresponden a alguien que no es nada creyente y de ideología de extrema derecha respectivamente.

La tabla $n^{\circ} 9$ resume los resultados del análisis discriminante aplicado a estas dos características personales para predecir el consumo de drogas.

1. Los dos indicadores entran en la función discriminante. La creencia religiosa es el indicador más relevante, tanto en el estudio español como en el estudio europeo.

2. La función discriminante resultante clasifica correctamente casi el $63 \%$ de los suje- 


\begin{tabular}{|c|c|c|}
\hline \multicolumn{3}{|c|}{$\begin{array}{l}\text { Tabla } n^{\circ} 8 \text { : Búsqueda de sensaciones y } \\
\text { el consumo o no consumo de drogas. }\end{array}$} \\
\hline \multicolumn{3}{|c|}{ CARACTERÍSTICAS PERSONALES } \\
\hline \multicolumn{3}{|c|}{ Búsqueda de sensaciones } \\
\hline Variable & Estudio español & Estudio europeo \\
\hline $\begin{array}{l}\text { Hacer cosas "locas", aunque } \\
\text { sean peligrosas }\end{array}$ & 0,95 & 0,96 \\
\hline Hacer lo que apetezca.. & 0,66 & 0,64 \\
\hline Algo peligroso... & n.s. & n.s. \\
\hline $\begin{array}{l}\text { Capacidad clasificatoria del modelo } \\
\text { que incluye los tres parámetros }\end{array}$ & $62 \%$ & $62,3 \%$ \\
\hline
\end{tabular}

\begin{tabular}{|c|c|c|}
\hline \multicolumn{3}{|c|}{$\begin{array}{c}\text { Tabla } n^{\circ} \text { 9: Creencias religiosas, ideología política } \\
\text { y el consumo o no consumo de drogas }\end{array}$} \\
\hline \multicolumn{3}{|c|}{ CARACTERÍSTICAS PERSONALES } \\
\hline \multicolumn{3}{|c|}{ Religión e Ideología política } \\
\hline Variable & Estudio español & Estudio europeo \\
\hline Religión & 0,94 & 0,98 \\
\hline Ideología & 0,56 & 0,35 \\
\hline $\begin{array}{l}\text { Capacidad clasificatoria del modelo } \\
\text { que incluye los dos parámetros }\end{array}$ & $62,4 \%$ & $62,6 \%$ \\
\hline
\end{tabular}

tos en su grupo de pertenencia, con resultados idénticos en ambos estudios.

Los consumidores de drogas se caracterizan por menor religiosidad y mayor inclinación hacia las ideologías "de izquierda" que los no-consumidores. Teniendo en cuenta las dos variables conjuntamente, la religiosidad resulta casi tres veces más relevante que la ideología a la hora de distinguir a los consumidores de los que no consumen drogas en el estudio europeo y casi el doble de relevante en el estudio español. La función que combina estas dos características personales también alcanza una capacidad moderada baja para predecir si un sujeto es consumidor o no (de modo similar al modelo basado en la búsqueda de sensaciones).

\subsection{Características personales y consumo de drogas}

Cada uno de los cuatro modelos anteriores nos permiten calcular para cada sujeto una probabilidad de ser consumidor de drogas. Estos cuatro atributos personales (desviación social, conductas de riesgo, búsqueda de sensaciones y religiosidad - ideología), han sido combinados en un modelo basado en el análisis discriminante, cuyos resultados globales están resumidos en la tabla $n^{\circ} 10$.

1. Estos cuatro indicadores de atributos personales quedan incluidos en el modelo europeo, mientras que en el modelo español queda excluida la búsqueda de sensaciones. La característica más relevante resulta ser en ambos modelos la realización de las conductas de riesgo en la conducción de vehículos, seguida de la desviación social, religiosidad e ideología.

2. Este modelo global basado en las características personales del entrevistado alcanza a clasificar correctamente alrededor de tres de cada cuatro sujetos en su grupo de pertenencia (consumidor o no consumidor). 


\begin{tabular}{|lcc|}
\hline \multicolumn{3}{|c|}{$\begin{array}{c}\text { Tabla } \mathbf{n}^{\mathbf{0}} \text { 10: Características personales y } \\
\text { el consumo o no consumo de drogas } \\
\text { CARACTERÍSTICAS PERSONALES }\end{array}$} \\
\hline \multicolumn{3}{|c|}{ Modelo global } \\
\hline Variable & Estudio español & Estudio europeo \\
\hline Conductas de riesgo & 0,86 & 0,79 \\
\hline Desviación social & 0,58 & 0,51 \\
\hline Religión e ideología & 0,41 & 0,49 \\
\hline Búsqueda de sensaciones & No incluido & 0,48 \\
\hline $\begin{array}{l}\text { Capacidad clasificatoria del modelo } \\
\text { que incluye los cuatro parámetros }\end{array}$ & $76,9 \%$ & $73,8 \%$ \\
\hline
\end{tabular}

De un modo resumido: ser una persona con un estilo de vida caracterizado por la inclinación hacia la conducta arriesgada y desvia$\mathrm{da}$, con bajas creencias religiosas y con orientación "de izquierdas" está sustancialmente asociado al consumo de drogas. Inversamente: no haber realizado nunca conductas de riesgo ni desviadas socialmente, mantener algún grado de creencias religiosas y una ideología política "de centro" o conservadora y no orientarse hacia la búsqueda desinhibida de riesgos y sensaciones está sustancialmente asociado con no ser consumidor de drogas.

En conclusión, nuestros resultados sobre la "personalidad" de los adolescentes y adultos jóvenes y su asociación con el uso o no uso de drogas vienen a confirmar los resultados hallados en los últimos estudios de Irefrea (Calafat et al., 1998, 1999, 2001) y apoyan sustancialmente las predicciones formuladas sobre del uso de drogas desde los modelos explicativos basados en la conducta - problema. Los factores estudiados se sitúan entre las características de personalidad que han sido consideradas como "factores de riesgo" en el uso de drogas, tras diversas revisiones de la literatura científica (Hawkins, Catalano y Miller, 1992; Petraitis, Flay y Miller, 1995; Becoña, 1999, Rhodes, Lilly, Fernández et al., 1999). Así pues, el uso de drogas en nuestra muestra se encuentra sustancialmente asociado al hecho de asumir valores socialmente no muy convencionales, mayor distanciamiento de los valores religiosos, mayor necesidad de independencia, conducta antisocial y búsqueda de sensaciones a través del riesgo y la conducta no convencional.

\section{GESTIÓN DE LA VIDA RECREATIVA DEL FIN DE SEMANA}

Los indicadores que definen la vida recreativa del sujeto en los estudios de encuestas de Irefrea se agrupan en tres áreas:

- La intensidad con que el sujeto se implica en la vida recreativa del fin de semana,

- La motivación para salir de marcha, y

- Los lugares preferidos por el sujeto para salir de marcha y donde se desarrolla su vida recreativa del fin de semana.

\subsection{Implicación en salir de marcha y con- sumo de drogas}

La implicación en salir de marcha se ha cuantificado a través de cinco indicadores, aunque los dos primeros se combinan para obtener uno solo:

1. El $n^{\circ}$ de fines de semana que el sujeto ha salido de marcha durante el último mes,

2. El $n^{\circ}$ de noches que el sujeto sale de marcha habitualmente por fin de semana. Estos dos primeros indicadores se combinan para obtener un indicador del $n^{\circ}$ de noches que el sujeto sale de marcha al mes. 
3. El $n^{\circ}$ de horas que habitualmente el sujeto dedica a salir de marcha.

4. El dinero total que el sujeto se gasta en salir de marcha durante un fin de semana en diversos conceptos, $y$

5. El porcentaje de dinero disponible que el sujeto se gasta en salir de marcha, agrupado en 4 categorías (menos del $25 \%$, del 25\%-50\%, del 50\%-75\% o más del $75 \%$ ).

La tabla $n^{\circ} 11$ resume los resultados más relevantes del análisis discriminante aplicado a estos indicadores de la implicación en salir de marcha:

1. Los cuatro indicadores han quedado incluidos en el modelo en ambos estudios. La función discriminante constituye un indicador positivo de la implicación en salir de marcha en cada sujeto y se asocia positivamente con la probabilidad de ser consumidor de drogas.

2. Las variables con mayor peso relativo en la función discriminante son en cierta medida similares en los estudios español y europeo: El $n^{\circ}$ de horas dedicadas a salir de marcha es la variable más discriminante en el estudio español. El resto de las variables están ordenadas del mismo modo en cuanto su relevancia en ambos estudios.

3. La función discriminante que combina estos cuatro indicadores clasifica correctamente el 69,2\% (algo más de dos tercios de los sujetos) en su grupo de pertenencia (consumidores o no consumidores de drogas) en el estudio europeo, y alcanza mayor capacidad clasificatoria en el estudio español (casi el 80\%).

Nuestros resultados demuestran que los sujetos que consumen drogas salen más fines de semana al mes, durante más noches cada fin de semana y durante más horas cada vez que salen que los que no consumen. Sin embargo, eso no significa que los no consumidores no salgan a divertirse: la gran mayoría salen dos o más fines de semana al mes, casi todos salen al menos una noche por fin de semana y lo más frecuente es que dediquen 6 horas a estos espacios de diversión cada vez que salen.

\subsection{Motivación para salir de marcha y consumo de drogas}

La motivación para salir de marcha se ha cuantificado a través de nueve indicadores (8 en el estudio español): Bailar, Conocer gente diferente, Reunirse con los amigos, Escuchar música, Buscar pareja, Buscar sexo, Romper la rutina diaria, Tomar alcohol (solo en el estudio europeo), Tomar drogas. Estos indicadores se evalúan en una escala de 4 puntos (desde $1=$ muy importante hasta $4=$ nada importante).

La tabla $n^{\circ} 12$ resume los resultados más relevantes del análisis discriminante aplicado

\begin{tabular}{|c|c|c|}
\hline \multicolumn{3}{|c|}{$\begin{array}{l}\text { Tabla } n^{\circ} 11: \text { Implicación en salir de marcha y } \\
\text { el consumo o no consumo de drogas }\end{array}$} \\
\hline \multicolumn{3}{|c|}{ SALIR DE MARCHA } \\
\hline \multicolumn{3}{|c|}{ Implicación en salir de marcha } \\
\hline Variable & Estudio español & Estudio europeo \\
\hline $\begin{array}{l}\text { Dinero que se gasta en salir durante } \\
\text { un fin de semana }\end{array}$ & 0,54 & 0,76 \\
\hline Porcentaje de dinero que se gasta en salir & 0,50 & 0,75 \\
\hline$N^{\circ}$ de noches que sale de marcha al mes & 0,45 & 0,44 \\
\hline $\begin{array}{l}N^{0} \text { de horas que sale de marcha } \\
\text { habitualmente }\end{array}$ & 0,68 & 0,44 \\
\hline $\begin{array}{l}\text { Capacidad clasificatoria del modelo } \\
\text { que incluye los cuatro parámetros }\end{array}$ & $78,1 \%$ & $69,2 \%$ \\
\hline
\end{tabular}




\begin{tabular}{|lcc|}
\hline \multicolumn{3}{|c|}{$\begin{array}{c}\text { Tabla } \mathbf{n}^{\circ} \\
\text { el consumo o no consumo de drogas } \\
\text { SALIR DE MARCHA }\end{array}$} \\
\hline \multicolumn{3}{|c|}{ Motivación para salir de marcha } \\
\hline Variable & Estudio español & Estudio europeo \\
\hline Tomar alcohol & N/A & 0,90 \\
\hline Tomar drogas & 0,98 & 0,70 \\
\hline Escuchar música & $n . S$. & 0,12 \\
\hline Buscar pareja & 0,05 & 0.06 \\
\hline Reunirse con los amigos & $-0,11$ &,- 01 \\
\hline Conocer gente diferente & 0,15 & $n . s$. \\
\hline Capacidad clasificatoria del modelo & $81,1 \%$ & $81 \%$ \\
\hline
\end{tabular}

a estos indicadores de la motivación para salir de marcha.

Los sujetos que consumen drogas otorgan más importancia a los ítems que son incluidos en el modelo con signo positivo, mientras que reunirse con los amigos es más importante para los que no consumen.

Los resultados más llamativos del modelo resultante son dos:

1. La vida recreativa no parece tener un significado notablemente distinto para consumidores y no consumidores de drogas en componentes tan esenciales como reunirse con los amigos, bailar, escuchar música, conocer gente o romper la rutina diaria, y que son los más importantes para ambos grupos. La oportunidad de consumir alcohol y drogas en un contexto recreativo que "regula" estos consumos parece ser un componente motivador específico para los consumidores de drogas.

2. Resulta llamativo que la motivación para salir de marcha alcance tan alta capacidad predictiva del consumo de drogas (cuatro quintas partes), mayor que las características personales (aislada y conjuntamente) comentadas anteriormente, y que la propia implicación en salir de marcha.

En resumen: el significado de la diversión para los consumidores no se distingue básicamente del significado que esta tiene para quienes no consumen. Más bien, resulta que los consumidores "añaden" al significado básico de la diversión del fin de semana la oportunidad de consumir alcohol y drogas, y es esta motivación "extra" para salir lo que distingue muy eficazmente a consumidores de no-consumidores.

\subsection{Lugares para salir de marcha y consu- mo de drogas}

Los lugares preferidos por el sujeto para salir de marcha y donde se desarrolla su vida recreativa nocturna se han definido operativamente a través de una escala de cuatro puntos que valora:

1. Su preferencia por lugares $\sin$ tabaco y sin alcohol (solo en el estudio europeo), sin drogas ilegales, y con bebidas no alcohólicas accesibles y baratas,

2. La frecuencia percibida de escenas violentas en estos lugares,

3. La accesibilidad percibida de lugares libres de drogas,

4. La preferencia por lugares con música muy alta, llenos de gente y de aspecto "descuidado",

5. La accesibilidad de preservativos en estos lugares, y

6. La limpieza de los espacios reservados para la higiene (servicios). 
La característica más relevante del contexto físico preferido para la diversión que distingue consumidores y no-consumidores está en la disponibilidad de drogas y en la existencia de modelos consumidores. Parece ser que los consumidores prefieren la existencia de tales modelos y la disponibilidad de estas sustancias para divertirse. Los no-consumidores demandan espacios recreativos libres de drogas, en mejores condiciones de comodidad y sonido. La preferencia por espacios recreativos de uno u otro tipo es capaz de clasificar casi cuatro de cada cinco entrevistados como consumidor o no de drogas.

\subsection{Modelo global relativo al salir de mar- cha y consumo de drogas}

El modelo basado en el análisis discriminante que reúne las tres funciones relativas al salir de marcha y su asociación con el consumo de drogas alcanza unos resultados que están resumidos en la tabla $n^{\circ} 14$.

1. Las tres funciones discriminantes estudiadas anteriormente son incluidas en el modelo en los dos estudios. La implicación en salir de marcha tiene una menor capacidad discriminativa en el estudio europeo, aunque su peso está más equilibrado en el estudio español. Los factores más relevantes en los estilos recreativos para discrimi- nar el consumo de drogas parecen ser la motivación a la hora de salir a divertirse y la preferencia por espacios recreativos con disponibilidad de sustancias y modelos consumidores.

2. La función discriminante que agrupa estos tres atributos de la vida recreativa del sujeto clasifica correctamente a casi 9 de cada 10 sujetos en su grupo de pertenencia (consumidor o no consumidor).

Lo que distingue a consumidores y no consumidores a la hora de gestionar su diversión no es tanto el tiempo que le dedican. Recordemos que el tiempo dedicado a salir de marcha tiene menor capacidad "discriminativa" que la motivación para salir y el contexto donde el sujeto se divierte. En lugar de esto, nuestros resultados demuestran que lo que distingue a los que consumen drogas de los que no lo hacen es el CONTEXTO donde desarrollan su vida recreativa del fin de semana (prefieren lugares con humo, drogas ilegales, de aspecto más bien descuidado, etc.) y el SIGNIFICADO que este ocio cobra para los primeros, donde el consumo de alcohol y drogas llega a convertirse en un componente que se añade a la diversión y socialización en su vida recreativa. Finalmente, resulta llamativo que con estos componentes de la vida recreativa del sujeto podamos "predecir" en

\begin{tabular}{|c|c|c|}
\hline \multicolumn{3}{|c|}{$\begin{array}{c}\text { Tabla } n^{\circ} 13: \text { Lugares preferidos para salir de marcha } \\
\text { y el consumo o no consumo de drogas }\end{array}$} \\
\hline \multicolumn{3}{|c|}{ SALIR DE MARCHA } \\
\hline \multicolumn{3}{|c|}{ Lugares para salir de marcha } \\
\hline Variable & Estudio español & Estudio europeo \\
\hline Lugares sin tabaco & N/A & 0,83 \\
\hline Lugares sin drogas ilegales & 0,91 & 0,73 \\
\hline Lugares de aspecto descuidado & $-0,35$ & $-0,31$ \\
\hline $\begin{array}{l}\text { Lugares con bebidas no alcohólicas } \\
\text { accesibles y económicas }\end{array}$ & 0,45 & 0,25 \\
\hline $\begin{array}{l}\text { Frecuencia de situaciones violentas } \\
\text { en estos lugares }\end{array}$ & n.s. & 0,03 \\
\hline Lugares con música no muy alta & 0,30 & n.s. \\
\hline Capacidad clasificatoria del modelo & $76,1 \%$ & $80 \%$ \\
\hline
\end{tabular}




\begin{tabular}{|c|c|c|}
\hline \multicolumn{3}{|c|}{$\begin{array}{l}\text { Tabla } n^{\circ} 14: \text { Estilos de salir de marcha y } \\
\text { el consumo o no consumo de drogas }\end{array}$} \\
\hline \multicolumn{3}{|c|}{ SALIR DE MARCHA } \\
\hline \multicolumn{3}{|c|}{ Modelo global } \\
\hline Variable & Estudio español & Estudio europeo \\
\hline Lugares para salir de marcha & 0,67 & 0,79 \\
\hline Motivación para salir de marcha & $-0,70$ & $-0,72$ \\
\hline Implicación en salir de marcha & 0,67 & 0,45 \\
\hline Capacidad clasificatoria del modelo & $85 \%$ & $86 \%$ \\
\hline
\end{tabular}

casi un $90 \%$ si el sujeto consume o no drogas, cuando otros factores tradicionalmente considerados "clave" (características de personalidad, contexto grupal y familiar, factores motivacionales y cognitivos, etc.) alcanzan menor capacidad "predictiva", como veremos durante este capítulo.

En consecuencia, nuestros resultados indican la necesidad de que las medidas preventivas y educativas en el uso y abuso de drogas incluyan la decodificación y construcción del significado que la diversión adquiere entre los adolescentes y jóvenes consumidores de drogas. Estas medidas deben analizar y poner de manifiesto los puntos débiles en los discursos que vinculan de modo inevitable la diversión con el uso y abuso de drogas legales e ilegales, para lo cual contamos con el ejemplo de muchos adolescentes y jóvenes que se implican activamente en la diversión del fin de semana sin recurrir al consumo. Simultáneamente, la construcción de una diversión sin el componente añadido del uso de drogas pasa por el desarrollo de la diversión en un entorno libre de drogas legales e ilegales, como una actividad normalizada más que nuestras comunidades ponen al alcance de los jóvenes.

\section{ENTORNO SOCIALY FAMILIAR}

En los estudios de encuestas de Irefrea en 1999 y 2001 se han incluido los siguientes cuatro constructos relativos al entorno social y familiar del sujeto:

1. Consumo de drogas entre familiares de primer grado,

2. Consumo de drogas entre los amigos,

3. Actitudes hacia el consumo de drogas entre los amigos, e

4. Integración social y familiar.

\subsection{Drogas en la familia y consumo de drofas en el sujeto}

Se ha evaluado el uso de alcohol, tabaco, cannabis y "otras" drogas ilegales en algún padre y hermano, utilizando una escala dicotómica (si / no). La tabla siguiente resume los resultados del modelo basado en la regresión logística:

1. El consumo de cannabis u otra droga ilegal en algún hermano resultan ser las variables más relevante y el consumo de alcohol o tabaco en alguno de los padres también entra en la función discriminante, aunque con menor relevancia.

2. La función discriminante clasifica correctamente a casi dos de cada tres sujetos en su grupo (consumidor o no consumidor) en ambos estudios.

Los consumos de alcohol, tabaco y drogas ilegales son más frecuentes entre familiares de los consumidores. Las variables incluidas en el modelo tienen una asociación más fuerte con el consumo de drogas en el sujeto, 


\begin{tabular}{|c|c|c|}
\hline \multicolumn{3}{|c|}{$\begin{array}{l}\text { Tabla } n^{\circ} 15: \text { Consumo de drogas en familiares de primer } \\
\text { grado y consumo o no consumo de drogas }\end{array}$} \\
\hline \multicolumn{3}{|c|}{ CONTEXTO FAMILIAR Y SOCIAL } \\
\hline \multicolumn{3}{|c|}{ Uso de drogas en la familia } \\
\hline Variable & Estudio español & Estudio europeo \\
\hline Algún hermano consume cannabis & 1,5 & 1,7 \\
\hline $\begin{array}{l}\text { Alguno de los padres consume } \\
\text { alcohol o tabaco }\end{array}$ & 0,9 & 0,8 \\
\hline Algún hermano consume otra ilegal & 1 & n.s. \\
\hline Constante & $-5,9$ & $-4,2$ \\
\hline Capacidad clasificatoria del modelo & $67,7 \%$ & $64,6 \%$ \\
\hline
\end{tabular}

explicando la asociación entre las demás variables y el consumo de drogas.

En resumen: la función que discrimina a los sujetos según su consumo / no consumo de drogas indica que los consumidores de drogas tienen mayor probabilidad de que alguno de sus hermanos consuma cannabis y otras drogas ilegales y de que alguno de los padres consuma alcohol o tabaco, siendo el consumo de drogas entre los hermanos notablemente más relevante que el consumo de drogas legales entre los padres.

Esta función discriminante alcanza aproximadamente la misma capacidad para "predecir" el consumo de drogas que la desviación social, algo más que la búsqueda de sensaciones y la religiosidad - ideología y algo menos capacidad que las conductas de riesgo comentadas anteriormente. En nuestros estudios, la capacidad "predictiva" del uso de drogas en la familia es inferior a la observada en los tres componentes asociados al salir de marcha.

\subsection{Drogas entre los amigos y consumo de drogas}

Se ha evaluado el uso de alcohol, alcohol en exceso, tabaco, cannabis, cocaína, éxtasis y "otras" drogas ilegales entre los amigos, utilizando una escala de cuatro puntos para describir la proporción de amigos que realizan frecuentemente estos consumos (desde ninguno hasta la mayoría). La tabla siguiente resume los resultados del modelo basado en el análisis discriminante:

1. De los 7 indicadores, 5 han sido incluidos en el modelo en ambos estudios y se refieren a consumos que son más frecuentes entre los amigos de los consumidores. Los consumos no incluidos en el modelo también son más frecuentes entre los amigos de los consumidores. Sin embargo, al ser incluidos en un modelo multivariante no forman parte de la función que distingue el consumo de drogas porque su asociación con éste es menor que la de las variables que sí entran en la función discriminante.

2. La variable más relevante resulta ser la proporción de amigos que consumen cannabis frecuentemente. Tras el consumo de cannabis, la proporción de amigos que se embriagan frecuentemente y que consumen frecuentemente drogas legales (alcohol y tabaco) y éxtasis muestran una relevancia muy similar entre si, aunque menor que el consumo frecuente de cannabis entre los amigos.

3. Estos 5 indicadores de consumo de drogas entre los amigos forman una función discriminante que clasifica correctamente a cuatro de cada cinco sujetos en su grupo (consumidor o no consumidor).

La capacidad de este modelo para distinguir consumidores / no consumidores es muy similar a lo observado en los dos principales componentes del salir de marcha (motivación y lugares preferidos), y superior a la 
alcanzada por el uso de drogas en la familia y los atributos relativos a la personalidad conjuntamente. En resumen, podemos acertar cuatro de cada cinco veces diciendo que un sujeto es consumidor o no de drogas en función de que tenga más o menos amigos consumidores frecuentes de cannabis, éxtasis, tabaco y alcohol.

\subsection{Actitudes hacia el consumo de drogas entre los amigos y consumo de drogas en el sujeto}

Se utilizó una escala con cuatro puntos que evaluaba el grado de acuerdo con afirmaciones relativas a actitudes ante en potencial consumo de drogas en una persona cercana (íntimo o pareja):

- Aceptación del amigo / amiga "como es", aunque consuma,
- Intentaría hacerle cambiar su actitud,

- Rompería la relación si siguiese consumiendo, y

- No iniciaría una relación con un consumidor.

La tabla $n^{\circ} 17$ resume los resultados del análisis discriminante aplicado a estos cuatro indicadores.

Los cuatro ítems resultan ser relevantes para distinguir una serie de actitudes entre consumidores y no consumidores. Los no consumidores manifiestan mayor rechazo a iniciar una relación íntima con un consumidor. En caso de iniciarla, los no consumidores manifiestan menor aceptación del consumo en el otro, mayor presión para que deje de consumir y mayor inclinación a finalizar la relación si ese cambio no se produce. En el estudio europeo, el factor más discriminativo es la actitud contraria a implicarse en relacio-

\begin{tabular}{|c|c|c|}
\hline \multicolumn{3}{|c|}{$\begin{array}{c}\text { Tabla } n^{\circ} 16: \text { Uso frecuente de drogas entre los amigos } \\
\text { y consumo o no consumo de drogas }\end{array}$} \\
\hline \multicolumn{3}{|c|}{ CONTEXTO FAMILIARY SOCIAL } \\
\hline \multicolumn{3}{|c|}{ Uso de drogas entre los amigos } \\
\hline Variable & studio español & Estudio europeo \\
\hline Amigos consumidores de cannabis & 0,93 & 0,94 \\
\hline Amigos consumidores de éxtasis & 0,48 & 0,54 \\
\hline Amigos fumadores & 0,55 & 0,52 \\
\hline Amigos consumidores de alcohol & 0,53 & 0,44 \\
\hline Amigos consumidores excesivos de alcoho & ol 0,64 & 0,43 \\
\hline Capacidad clasificatoria del modelo & $82,3 \%$ & $81 \%$ \\
\hline
\end{tabular}

\begin{tabular}{|c|c|c|}
\hline \multicolumn{3}{|c|}{$\begin{array}{l}\text { Tabla } n^{\circ} \text { 17: Actitudes de aceptación o rechazo del uso de } \\
\text { drogas en amigos íntimos y consumo o no consumo de drogas }\end{array}$} \\
\hline \multicolumn{3}{|c|}{ CONTEXTO FAMILIAR Y SOCIAL } \\
\hline \multicolumn{3}{|c|}{ Actitudes hacia el uso de drogas en un amigo / a } \\
\hline Variable & Estudio español & Estudio europeo \\
\hline No se implicaría con un consumidor/a & 0,48 & 0,90 \\
\hline Rompería la relación si persiste & 0,40 & 0,73 \\
\hline Le aceptaría & $-0,80$ & $-0,68$ \\
\hline Intentaría cambiar su actitud & 0,67 & 0,49 \\
\hline Capacidad clasificatoria del modelo & $68,4 \%$ & $66,7 \%$ \\
\hline
\end{tabular}


nes íntimas con consumidores. En el estudio realizado en las cuatro ciudades españolas las actitudes más discriminativas son la aceptación del consumo en una persona muy cercana y los intentos por cambiar la actitud del otro (una vez que la relación se ha iniciado).

La capacidad de este modelo para "discriminar o predecir" es moderada en ambos estudios, comparable al consumo de drogas entre los familiares y el modelo de características personales, y menor que la alcanzada por las variables relativas al salir de marcha y el consumo de drogas entre los amigos.

\subsection{Integración social y familiar y consu- mo de drogas}

Se utilizó una escala con cuatro puntos que evaluaba el grado de acuerdo - desacuerdo con 10 afirmaciones que indicaban:

- La familia le permite tomar parte en las decisiones familiares,

- Tiene facilidad para hacer nuevos amigos, para relacionarse con el otro sexo, sus opiniones son valoradas positivamente por sus amigos y le gusta estar solo (solo en el estudio europeo).

- Siente agrado por las actividades cotidianas (estudio / trabajo), por compartir tareas domésticas con la familia y por pasar momentos con la familia.

- Participa en actividades sociales o voluntariado, y desea contribuir a un mundo mejor.

La tabla $n^{0} 18$ resume los resultados del análisis discriminante aplicado a estos indicadores.

En el estudio europeo, los no consumidores se implican más en actividades altruistas, en las interacciones y decisiones familiares y en las actividades cotidianas, siendo estas características las más relevantes a la hora de distinguirles de los consumidores, quienes parecen percibirse como más hábiles para las interacciones heterosexuales. En el estudio español, los factores discriminativos relevantes son únicamente un mayor agrado por las actividades en familia y el deseo de contribuir a mejorar el mundo.

La capacidad de los dos modelos para "predecir" y discriminar el consumo de drogas es moderado o bajo, y menor en ambos estudios que la alcanzada por las conductas de riesgo, los diferentes indicadores de la vida recreativa y el consumo de drogas entre los amigos.

\begin{tabular}{|c|c|c|}
\hline \multicolumn{3}{|c|}{$\begin{array}{c}\text { Tabla } n^{\circ} \text { 18: Integración social y familiar y consumo } \\
\text { o no consumo de drogas }\end{array}$} \\
\hline \multicolumn{3}{|c|}{ CONTEXTO FAMILIAR Y SOCIAL } \\
\hline \multicolumn{3}{|c|}{ Integración social y familiar } \\
\hline Variable & studio español & Estudio europeo \\
\hline Participa en voluntariado & n.s. & 0,56 \\
\hline $\begin{array}{l}\text { Comparte momentos agradables } \\
\text { con la familia }\end{array}$ & 0,79 & 0,53 \\
\hline Disfruta con las actividades cotidianas & n.s. & 0,49 \\
\hline Le resulta fácil relacionarse con el otro sex & exo N/A & $-0,38$ \\
\hline Participa en decisiones familiares & n.s. & 0,35 \\
\hline $\begin{array}{l}\text { Sus opiniones son importantes } \\
\text { para sus amigos }\end{array}$ & N/A & $-0,16$ \\
\hline Participa en las tareas domésticas & n.s. & 0,11 \\
\hline Desea contribuir a un mundo mejor & 0,77 & n.s. \\
\hline Capacidad clasificatoria del modelo & $60 \%$ & $62 \%$ \\
\hline
\end{tabular}




\subsection{Entorno social y familiar y consumo de drogas: modelo global}

Las funciones discriminantes y de regresión relativas al consumo de drogas en la familia, el consumo frecuente de drogas en los amigos, la actitud hacia el consumo en un amigo íntimo y la integración social y familiar (como han sido descritas previamente) son incluidas en el modelo global. La tabla $n^{\circ} 19$ resume los resultados del análisis discriminante aplicado a distinguir consumidores de no consumidores:

En el estudio europeo las cuatro funciones discriminantes comentadas anteriormente por separado juegan un papel relevante para distinguir el consumo de drogas, mientras que el modelo únicamente español no incluye las actitudes hacia el consumo de drogas en una relación muy cercana. Según lo esperable, la variable más relevante resulta ser el consumo frecuente de drogas entre los amigos.

Podemos acertar cuatro de cada cinco veces en predecir si un sujeto es o no consumidor de drogas en la medida que tenga más o menos amigos consumidores de cannabis, éxtasis y drogas legales y que acepte el consumo de drogas en una pareja o amigo íntimo (especialmente en el ámbito europeo). Nos puede ayudar en esta predicción también tener en cuenta si tiene o no algún hermano consumidor de cannabis, si alguno de sus padres consume o no alcohol, y si tiene un nivel menor o mayor de satisfacción con su vida cotidiana y con sus interacciones familiares y se implica más o menos en actividades altruistas. Finalmente, cabe reseñar que el modelo basado en el contexto social y familiar no alcanza la capacidad "discriminativa o predictiva" observada en el modelo relativo a salir de marcha, a pesar de que habitualmente se le considera como uno de los factores más claves a la hora de predecir el consumo de drogas.

En este apartado relativo al entorno social y familiar se encuentra, una vez más, evidencia que muestra la relevancia clave de estos factores en el uso y abuso recreativo de drogas. El uso frecuente de drogas no se desarrolla y mantiene aisladamente del entorno del adolescente y joven, sino que en la mayoría de los casos se convierte en si misma en un criterio para la inclusión y exclusión activa en la red social y en un componente de la identidad psicosocial del sujeto. La literatura científica que establece las bases teóricas de la prevención en el uso de drogas y diversas revisiones ya han establecido la relevancia del uso de drogas en los pares y familiares como factor de riesgo en el uso y abuso de drogas (Becoña, 1999; Rhodes et al., 1999). El proceso de incluir el uso de drogas como un componente de la identidad grupal y personal no está presente entre los no-consumidores, lo que constituye un elemento discri-

\begin{tabular}{|c|c|c|}
\hline \multicolumn{3}{|c|}{$\begin{array}{l}\text { Tabla } n^{\circ} 19: \text { Contexto social y familiar y el } \\
\text { consumo o no consumo de drogas }\end{array}$} \\
\hline \multicolumn{3}{|c|}{ CONTEXTO FAMILIAR Y SOCIAL } \\
\hline \multicolumn{3}{|c|}{ Modelo global } \\
\hline Variable & Estudio español & Estudio europeo \\
\hline $\begin{array}{l}\text { Consumo frecuente de drogas } \\
\text { entre los amigos }\end{array}$ & 0,96 & 0,95 \\
\hline $\begin{array}{l}\text { Actitudes hacia el consumo de drogas } \\
\text { en alguien muy cercano }\end{array}$ & n.s. & $-0,44$ \\
\hline $\begin{array}{l}\text { Consumo de drogas en algún familiar } \\
\text { de primer grado }\end{array}$ & 0,46 & 0,43 \\
\hline Integración social y familiar & $-0,27$ & $-0,32$ \\
\hline Capacidad clasificatoria del modelo & $84 \%$ & $82,6 \%$ \\
\hline
\end{tabular}


minativo y posiblemente un componente preventivo.

Nuestro estudio también pone de manifiesto la relevancia que tienen algunos aspectos de la vida social y familiar a la hora de predecir del uso de drogas. Nuestros resultados vienen a coincidir en alguna medida con las afirmaciones de algunos autores que consideran al uso de drogas como parte de un conjunto de comportamientos asociados a su vez con valores intra-personales (que se orientan más hacia el interés personal). Inversamente, los no consumidores mantendrían según estos autores una jerarquía de valores caracterizada por una mayor importancia relativa hacia valores interpersonales o sociales, orientación hacia otros significativos y hacia otras áreas vitales valiosas para el sujeto, incluyendo la conducta pro-social y la autopercepción o "self" (Peele, 1987; Rokeach, 1979; Pettet, 1993).

\section{ACTITUDES, PERCEPCIÓN DE RIESGOS $Y$ EXPECTATIVAS RELACIONADAS CON LAS DROGAS}

En los dos estudios de encuestas se han incluido ítems relativos a los siguientes cinco constructos:
1. Percepción de los motivos por los que algunas personas no consumen drogas,

2. Percepción de los motivos por los que algunas personas sí consumen drogas cuando salen a divertirse,

3. Percepción de la imagen que tienen los que consumen drogas de los que no las consumen,

4. Actitudes hacia el control social y legal de las drogas, y

5. Percepción de los riesgos asociados al consumo de drogas.

\subsection{Motivos para no consumir drogas}

Se preguntaba al sujeto acerca de su grado de acuerdo o desacuerdo con 10 afirmaciones que expresaban motivos por los que algunas personas no consumen drogas legales ni ilegales. La tabla $n^{\circ} 20$ resume los resultados del análisis discriminante aplicado a estos motivos con el fin de distinguir consumidores de no consumidores.

Los resultados alcanzados por el análisis discriminante podrían resumirse en los siguientes puntos:

1. Entre los 7 motivos incluidos en la función discriminante, los no consumidores consideran el consumo de drogas más falto de interés en si mismo, que solo es propio de

\begin{tabular}{|c|c|c|}
\hline \multicolumn{3}{|c|}{$\begin{array}{l}\text { Tabla } n^{\circ} \text { 20: Percepción de los motivos para no } \\
\text { consumir drogas y consumo o no consumo de drogas }\end{array}$} \\
\hline \multicolumn{3}{|c|}{ ACTITUDES, PERCEPCIÓN DE RIESGOS Y EXPECTATIVAS } \\
\hline \multicolumn{3}{|c|}{ Percepción de los motivos para no consumir drogas } \\
\hline Variable & Estudio español & Estudio europeo \\
\hline $\begin{array}{l}\text { Las drogas son solamente para quienes } \\
\text { no saben lo que quieren }\end{array}$ & s $\quad 0,69$ & 0,63 \\
\hline Porque aún no las han probado & $-0,67$ & $-0,57$ \\
\hline Porque el mundo sería mejor sin drogas & 0,29 & 0,38 \\
\hline Porque no están interesados en ellas & $-0,15$ & 0,32 \\
\hline Porque sus familiares lo desaprueban & n.s. & $-0,20$ \\
\hline Porque consumir drogas es costoso & n.s. & 0,18 \\
\hline Porque temen convertirse en adictos & n.s. & $-0,18$ \\
\hline Capacidad clasificatoria del modelo & $71,7 \%$ & $68 \%$ \\
\hline
\end{tabular}


las vidas "sin sentido" y que empeora la situación de la comunidad global. Inversamente, los consumidores argumentan que no se consumen drogas por desconocimiento de sus efectos y "por miedo a efectos negativos del consumo".

2. Estas percepciones de los motivos que llevan a no consumir resultan relevantes para "predecir" y "discriminar" consumidores de no consumidores (alrededor del 70\%). Su capacidad es comparable a la alcanzada por la desviación social, las conductas de riesgo y la implicación en salir de marcha, más relevante que la búsqueda de sensaciones, religiosidad - ideología y que el consumo de drogas en la familia y la integración social y familiar. La capacidad de distinguir el consumo es claramente menor que la del consumo de drogas entre los amigos y la motivación y entorno de la vida recreativa.

\subsection{Motivos para si consumir drogas}

Se preguntaba al sujeto acerca de su grado de acuerdo o desacuerdo con diversas afirmaciones que expresaban motivos por los que algunas personas sí consumen drogas legales e ilegales cuando salen a divertirse los fines de semana. La tabla $n^{\circ} 21$ resume los resultados del análisis discriminante aplicado a estos motivos con el fin de distinguir consumidores de no consumidores.
La mayoría de los consumidores están de acuerdo en afirmar que el consumo de drogas les resulta útil para sus metas en una amplia gama de situaciones, mientras que generalmente son la mitad o menos los noconsumidores que están de acuerdo en señalar que el consumo de drogas les sirve a quienes lo hacen para alcanzar sus metas en tales situaciones.

Los resultados en este apartado son parcialmente coincidentes en ambos estudios: el factor más discriminativo es el valor instrumental atribuido al consumo de drogas para mejorar la experiencia vital. El modelo resultante en el estudio español alcanza una capacidad discriminativa ligeramente superior a la observada en el estudio europeo.

Podemos predecir y acertar dos de cada tres veces o más si decimos que un sujeto en estas dos muestras es consumidor o no de drogas si considera o no que las drogas mejoran la experiencia vital, ayudan a experimentar la música y el baile, a divertirse y a sentirse mejor.

\subsection{Imagen social del no-consumidor de drogas}

En los dos estudios de encuestas también se preguntaba acerca del grado de acuerdo o desacuerdo con 7 afirmaciones sobre la imagen que los consumidores tienen de quienes

\begin{tabular}{|c|c|c|}
\hline \multicolumn{3}{|c|}{$\begin{array}{l}\text { Tabla } n^{\circ} \text { 21: Percepción de los motivos para sí consumir } \\
\text { drogas y el consumo o no consumo de drogas }\end{array}$} \\
\hline \multicolumn{3}{|c|}{ ACTITUDES, PERCEPCIÓN DE RIESGOS Y EXPECTATIVAS } \\
\hline \multicolumn{3}{|c|}{ Percepción de los motivos para SI consumir drogas } \\
\hline Variable & Estudio español & Estudio europeo \\
\hline $\begin{array}{l}\text { Ayudan a tener una experiencia vital } \\
\text { más completa }\end{array}$ & 0,77 & 0,74 \\
\hline Ayudan a experimentar la música y el bail & 0,55 & 0,72 \\
\hline Ayudan a sentirse mejor & n.s. & 0,66 \\
\hline Puede resultar placentero & 0,76 & n.s. \\
\hline Ayudan a divertirse & 0,62 & n.s. \\
\hline Ayudan a evadirse de los problemas & n.s. & 0,04 \\
\hline Capacidad clasificatoria del modelo & $72,8 \%$ & $67,6 \%$ \\
\hline
\end{tabular}


no consumen drogas legales ni ilegales. La tabla $n^{\circ} 22$ resume los resultados del análisis discriminante aplicado a estos componentes de la percepción social de los no-consumidores.

En ambos estudios, la capacidad clasificatoria del modelo es moderada - baja. Las diferencias en el estudio europeo indican que los no-consumidores son percibidos por los consumidores como personas más aisladas socialmente, que se divierten menos y son menos conflictivos, mientras que los consumidores resaltan menos estos componentes de la imagen social de aquellos. En el estudio español, las diferencias consisten en que los no-consumidores se sienten percibidos como más respetados, menos conflictivos, algo "raros" y menos divertidos, mientras que los consumidores resaltan menos estos componentes en la imagen social de los primeros.

\subsection{Actitudes hacia la prevención y control legal de las drogas}

Los sujetos entrevistados indicaban su grado de acuerdo / desacuerdo con 7 afirmaciones relativas a la prevención y control social de las drogas. Los consumidores de drogas se distinguen de los no consumidores por una actitud menos favorable hacia una legislación estricta en materia de alcohol y drogas en general y de las drogas ilegales en particular. Su actitud también es menos desfavorable hacia la restricción de la venta de alcohol a menores, hacia el castigo del consumo público de drogas ilegales y alcohol, y tienen un menor grado de información acerca de la legislación sobre alcohol y drogas.

La tabla $n^{\circ} 23$ resume los resultados del análisis discriminante aplicado a estas actitudes hacia el control social de las drogas:

Al introducirse conjuntamente en el modelo discriminante, en ambos estudios resultan funciones discriminantes que clasifican correctamente alrededor de tres de cada cuatro sujetos. Su capacidad predictiva es mayor que la del consumo de drogas en la familia, las actitudes hacia el consumo de drogas en una persona muy cercana, la integración social y familiar y que los demás factores motivacionales y cognitivos comentados en puntos anteriores. Sin embargo, no llegan a ser tan capaces de discriminar el consumo de drogas como el consumo de drogas entre los amigos y los componentes más relevantes del salir de marcha.

\subsection{Percepción de los riesgos asociados al consumo de drogas}

Los sujetos entrevistados indicaban su percepción del grado de peligrosidad asociado a una serie de patrones de consumo de drogas legales e ilegales. La tabla siguiente resume los resultados del análisis discriminante aplicado a estas percepciones del riesgo asociado al consumo de drogas:

\begin{tabular}{|c|c|c|}
\hline \multicolumn{3}{|c|}{$\begin{array}{l}\text { Tabla } n^{\circ} 22 \text { : Percepción de la imagen del no-consumidor de drogas } \\
\text { por quienes consumen y su asociación con el consumo de drogas }\end{array}$} \\
\hline \multicolumn{3}{|c|}{ ACTITUDES, PERCEPCIÓN DE RIESGOS Y EXPECTATIVAS } \\
\hline \multicolumn{3}{|c|}{ Imagen social de los no consumidores } \\
\hline Variable & Estudio español & Estudio europeo \\
\hline Se divierten menos & 0,39 & 0,78 \\
\hline Tienen menos amigos & n.s. & 0,77 \\
\hline Son más respetados & 0,72 & n.s. \\
\hline Son menos conflictivos & 0,52 & 0,56 \\
\hline Son vistos como "raros" & 0,43 & n.s. \\
\hline Capacidad clasificatoria del modelo & $61,2 \%$ & $64,1 \%$ \\
\hline
\end{tabular}




\begin{tabular}{|c|c|c|}
\hline \multicolumn{3}{|c|}{$\begin{array}{c}\text { Tabla } n^{\circ} 23 \text { : Actitudes hacia el control social de las drogas } \\
\text { y consumo o no consumo de drogas }\end{array}$} \\
\hline \multicolumn{3}{|c|}{ ACTITUDES, PERCEPCIÓN DE RIESGOS Y EXPECTATIVAS } \\
\hline \multicolumn{3}{|c|}{ Actitudes hacia el control social de las drogas } \\
\hline Variable & studio español & Estudio europeo \\
\hline $\begin{array}{l}\text { La legislación sobre drogas debería } \\
\text { ser menos estricta }\end{array}$ & 0,80 & 0,79 \\
\hline $\begin{array}{l}\text { El uso público de drogas ilegales } \\
\text { debería ser penalizado }\end{array}$ & $-0,84$ & $-0,70$ \\
\hline $\begin{array}{l}\text { El uso público de alcohol en la calle } \\
\text { debería ser penalizado }\end{array}$ & $-0,62$ & $-0,53$ \\
\hline El consumo de drogas debe estar regulad & 0,57 & n.s. \\
\hline $\begin{array}{l}\text { Grado de información acerca de la } \\
\text { legislación sobre drogas }\end{array}$ & 0,19 & 0,24 \\
\hline Capacidad clasificatoria del modelo & $76,4 \%$ & $73 \%$ \\
\hline
\end{tabular}

Los resultados de ambos estudios son similares, en cuanto al peso relativo de las variables que entran en la función discriminante y a la capacidad predictiva del modelo resultante. En un grupo similar a nuestras muestras, podemos predecir acertadamente en tres de cada cuatro predicciones si un sujeto consume o no drogas en función de si percibe como menos o más peligroso consumir cannabis frecuentemente, consumir alcohol hasta la intoxicación o consumir cocaína ocasionalmente.

\subsection{Actitudes, precepción de riesgos y expectativas: predicción del uso de drogas}

Las cinco funciones discriminantes expuestas en esta sección fueron introducidas conjuntamente en un nuevo análisis discriminante para distinguir a los consumidores de drogas de los no-consumidores. La tabla $n^{\circ}$ 24 muestra los resultados obtenidos:

1. Nuevamente, se observa que los resultados hallados en los dos estudios (español y europeo) son notablemente similares. Los cinco conceptos resultan relevantes para distinguir a los sujetos según consuman o no drogas:

a. Las actitudes hacia el control social y legal de las drogas y la percepción de riesgos asociados al consumo son los más relevan- tes para diferenciar sujetos según su grupo.

b. La percepción de los motivos que tienen muchos adolescentes y jóvenes para no consumir drogas y los motivos que llevan a algunos sujetos a consumir drogas al salir de marcha alcanzan una relevancia intermedia para clasificar a los sujetos en su grupo (consumidor / no consumidor).

c. La imagen percibida del no consumidor entre quienes consumen drogas resulta menos relevante que los otros 4 constructos.

2. Globalmente, esta función discriminante que agrupa percepciones, expectativas y actitudes hacia las drogas es capaz de clasificar correctamente a cuatro de cada cinco sujetos, mayor capacidad que las características personales y ligeramente menor que el contexto familiar - social y que el modelo sobre la gestión del salir de marcha.

La utilidad principal de este modelo consiste en que está formado por variables dinámicas y susceptibles de modificación a través de la educación e intervenciones preventivas. Comentaremos la posible utilidad para la educación y prevención del uso de drogas que encontramos en nuestros resultados sobre los dos conceptos más relevantes de los que 


\begin{tabular}{|lcc|}
\hline \multicolumn{2}{|c|}{$\begin{array}{c}\text { Tabla } \mathbf{n}^{\circ} \text { 24: Percepción de los riesgos de consumir } \\
\text { drogas y consumo o no consumo de drogas } \\
\text { ACTITUDES, PERCEPCIÓN }\end{array}$} \\
\hline \multicolumn{3}{|c}{ Percepción de los riesgos asociados al consumo } \\
\hline Variable & Estudio español & Estudio europeo \\
\hline Consumir regularmente cannabis & 0,75 & 0,86 \\
\hline Embriagarse frecuentemente & 0,67 & 0,54 \\
\hline Cuatro bebidas alcohólicas & 0,66 & 0,51 \\
\hline Consumir cocaína mensualmente & 0,64 & 0,50 \\
\hline Fumar un paquete de cigarillos diario & n.s. & 0,19 \\
\hline Capacidad clasificatoria del modelo & $75 \%$ & $72,4 \%$ \\
\hline
\end{tabular}

\begin{tabular}{|c|c|c|}
\hline \multicolumn{3}{|c|}{$\begin{array}{l}\text { Tabla } n^{\circ} \text { 25: Percepciones, expectativas y actitudes sobre } \\
\text { el uso de drogas y consumo o no consumo de drogas }\end{array}$} \\
\hline \multicolumn{3}{|c|}{ ACTITUDES, PERCEPCIÓN DE RIESGOS Y EXPECTATIVAS } \\
\hline \multicolumn{3}{|c|}{ Modelo global } \\
\hline Variable & studio español & Estudio europeo \\
\hline Control social de las drogas & 0,74 & $-0,72$ \\
\hline Percepción de riesgos asociados al consum & no 0,69 & 0,72 \\
\hline Motivos para no consumir & 0,66 & 0,57 \\
\hline Motivos para sí consumir & $-0,63$ & $-0,53$ \\
\hline Imagen social del no consumidor & 0,30 & 0,39 \\
\hline Capacidad clasificatoria del modelo & $82,3 \%$ & $80,8 \%$ \\
\hline
\end{tabular}

forman el modelo: a) las actitudes hacia el control y la percepción de los riesgos asociados al consumo; b) la motivación para consumir o no consumir drogas.

El resultado más relevante hallado en este apartado se refiere a la actitud de los consumidores contraria al control social del uso de drogas y su baja percepción del riesgo asociado al consumo de drogas legales e ilegales. Los sujetos que utilizan drogas ilegales son generalmente conscientes de muchos efectos nocivos de las drogas (por ejemplo, que podían disminuir la capacidad para la conducción), y los consumidores de nuestra muestra afirman que prevendrían a un amigo en contra de conducir bebido y están a favor de penalizar conducción bajo los efectos del alcohol. Al mismo tiempo, muchos de estos sujetos reconocen que el consumo de drogas está claramente asociado a la conducción: conducen para adquirir las drogas, consumen frecuentemente dentro de los vehículos, se interesan menos por la ilegalidad de su comportamiento y en bastantes casos han conducido sin licencia. Este fenómeno de percibir el riesgo de usar drogas para uno mismo como menor de lo que se percibe para otros es denominado "sesgo optimista" o "sentimiento de invulnerabilidad" (Leigh, 1999) y está presente entre muchos jóvenes consumidores. Coincidiendo con nuestros resultados, existe evidencia acumulativa demostrando que los consumidores de drogas tienden a infravalorar las consecuencias negativas del consumo y la probabilidad de riesgo (Aitken, Kerger y Crofts, 2000), especialmente una especie de "miopía temporal" que consiste en negar sus consecuencias a largo plazo.

Aunque la percepción de riesgos y la vulnerabilidad al daño son aspectos centrales en muchas de las teorías psicológicas sobre la conducta de riesgo (Cummings, Becker y 
Maile, 1980; Rogers, 1984; Weinstein, 1993), aún resulta difícil explicar los factores que determinan la baja percepción del riesgo asociado a una conducta arriesgada. Los psicólogos cognitivos e investigadores sobre los procesos de decisión han puesto de manifiesto una serie de impedimentos que dificultan una toma de decisiones racional (Leigh, 1999). La capacidad de las personas para calcular el riesgo es pobre. Más aún, la gente a menudo infravalora la propia vulnerabilidad a una variedad de eventos desgraciados, incluidas las consecuencias dañinas del consumo de alcohol y drogas. Esta tendencia es aún más fuerte para los eventos más estigmatizados y para los resultados que se suponen más controlables, y las consecuencias del consumo de alcohol y drogas reúnen ambas características. Estos sesgos y déficit en la percepción de los riesgos asociados al uso de drogas pueden ser también consecuencia de distorsiones en el procesamiento de la información y estar afectadas por el consumo regular de alcohol y otras drogas. Además, parecen ser resistentes al cambio a través de la exposición a intervenciones educativas sobre drogas.

La percepción del riesgo asociado al consumo de drogas está también en la base de una actitud más o menos favorable hacia el control social del consumo, al menos en una buena parte de los consumidores. Aunque no parece fácil conseguir aumentar la percepción de los riesgos asociados a las drogas entre adolescentes y jóvenes, esta tarea constituye un desafío para la prevención del uso de drogas. Otras evidencias mostradas en este y otros capítulos indican que una buena parte de los consumidores de drogas también muestran mayor inclinación hacia las conductas de riesgo asociadas a la denominada "delincuencia de baja intensidad" (hurtos, vandalismo, violencia, etc.) y a la búsqueda de sensaciones asociadas a diversas conductas arriesgadas. En estos, una baja percepción de los riesgos asociados al consumo de drogas y una actitud contraria hacia el control social de las drogas a través de normas reguladoras estaría asociada, con una inclinación hacia la conducta - problema en general (Jessor, 1993; Jessor et al., 1995). En estos consumidores, aumentar la percepción de los riesgos asociados al consumo de drogas y aceptar el control social de este consumo requiere modificar una pauta generalizada favorable al riesgo y que forma parte de un estilo de vida.

Un segundo bloque de resultados relevantes se refieren a la motivación para consumir y para no consumir drogas, tal como es percibida por consumidores y no consumidores. La comprensión de la motivación para consumir o no consumir drogas puede ser útil para la lógica preventiva. Los mensajes preventivos orientados a impedir la iniciación, experimentación y evitación de los riesgos y consecuencias del abuso "encajan" mejor dentro de la lógica de los consumidores para no consumir drogas, aunque (en su caso) ese discurso no parece haber sido lo suficientemente eficaz. Inversamente, el "discurso preventivo" que caracteriza a los no consumidores se dirige hacia la propia falta de sentido y utilidad del consumo en sus vidas y en la mejora del mundo en que desean vivir. Tal vez, el mensaje que subyace es que la prevención debería orientar más sus objetivos hacia resaltar a los no consumidores como modelos de comportamiento, quienes, además de mantener una actitud de control y rechazo del consumo, mantienen sus objetivos vitales centrados en ámbitos de actividad alejados y sustancialmente incompatibles con el uso de drogas. El análisis de este estilo de vida donde el consumo no tiene sentido ni cabida podría presentarse como elemento de comparación al estilo de vida de muchos consumidores: ¿qué entienden unos y otros por una vida "más plena", y porqué las drogas son necesarias o útiles según los consumidores para alcanzarla?, ¿qué entienden unos y otros por "bienestar," y porque las drogas son útiles o necesarias para alcanzarlo, según los consumidores?, ¿qué entienden unos y otros por experimentar la música y el baile, y porqué solo algunos parecen necesitar las drogas para ello?, etc. Tal vez, una parte de la respuesta está en la orientación inmediata de muchos consumidores hacia los riesgos, la sobre-estimulación y búsqueda de sensacio- 
nes, la desinhibición y los valores menos convencionales.

\section{MODELO GLOBAL Y PREDICCIÓN DEL USO DE DROGAS}

Con el fin de valorar el peso relativo de los modelos anteriores conjuntamente se construyó un modelo discriminante final que agrupa los diferentes conjuntos de variables. La tabla $n^{\circ} 26$ muestra los resultados relativos a este modelo final:

1. Las cuatro funciones discriminantes realizan una aportación relevante para distinguir a los sujetos según su consumo de drogas en el estudio europeo, mientras que las características personales no entran en el modelo resultante en el estudio español. El peso relativo de las variables introducidas en el modelo se ordenan de igual modo en ambos estudios:

a. La gestión de la vida recreativa del fin de semana (lugares preferidos, la motivación y la implicación para salir de marcha) forma el componente más distintivo.

b. El contexto familiar y social (consumo frecuente de drogas entre los amigos y familiares, la aceptación de este consumo y la integración social y familiar) resulta ser el segundo componente del modelo en relevancia. c. Las actitudes hacia el control social y legal de las drogas, percepción de riesgos, motivaciones para consumir y no consumir drogas y la imagen del no consumidor forman un tercer componente del modelo, menos relevante que los dos anteriores.

d. Las características relativas a la personalidad son menos relevantes en el modelo global que las tres funciones anteriores, y solamente resultan relevantes en el estudio europeo.

2. La capacidad del modelo de distinguir el grupo de pertenencia (consumidor / no consumidor) puede considerarse alta: casi 9 de cada 10 sujetos son "clasificados" correctamente en su grupo.

\section{CONCLUSIONES}

El capítulo compara a quienes consumen o no consumen drogas legales e ilegales, en dos muestras: una de ellas formada por 806 jóvenes y adolescentes entrevistados por Irefrea en el año 1999 en 4 ciudades españolas y otra formada por 1777 jóvenes y adolescentes entrevistados por Irefrea en el año 2001 en 10 ciudades europeas. Ambas muestras están balanceadas por género y grupo de edad (adolescentes y adultos jóvenes).

Las variables estudiadas se agrupan en cuatro áreas: contexto familiar y amigos, características relativas a la personalidad, varia-

\begin{tabular}{|c|c|c|}
\hline \multirow{2}{*}{\multicolumn{3}{|c|}{$\begin{array}{c}\text { Tabla }^{\circ}{ }^{\circ} \text { 26: Modelo global resultante del estudio y e } \\
\text { consumo o no consumo de drogas } \\
\text { MODELO GLOBAL }\end{array}$}} \\
\hline & & \\
\hline Variable & Estudio español & Estudio europeo \\
\hline Salir de marcha & 0,83 & 0,85 \\
\hline Entorno social y familiar & $-0,80$ & $-0,75$ \\
\hline $\begin{array}{l}\text { Actitudes, percepción y } \\
\text { expectativas }\end{array}$ & 0,74 & 0,66 \\
\hline Características personales & n.s. & $-0,47$ \\
\hline $\begin{array}{l}\text { Capacidad clasificatoria del } \\
\text { modelo }\end{array}$ & $89,6 \%$ & $88,3 \%$ \\
\hline
\end{tabular}


bles motivacionales y cognitivas relativas a las drogas (actitudes, percepciones, expectativas y motivación) y gestión de la vida recreativa del fin de semana. Las cuatro áreas estudiadas y la mayoría de las variables incluidas en cada una de ellas son relevantes para distinguir a los que consumen drogas legales e ilegales de los que no lo hacen. Se ha construido un modelo global basado en los análisis discriminantes para distinguir ambos grupos, que combina estas cuatro áreas y sugiere que los estilos de salir el fin de semana son los más relevantes en la predicción del consumo de drogas, junto con el factor relativo al consumo de drogas en los pares y familiares. El modelo clasifica correctamente casi el $90 \%$ de los sujetos en consumidores o no consumidores, independientemente del género y grupo de edad.

Se sugiere que las intervenciones educativas y la prevención deben tener en cuenta la gestión del ocio del fin de semana y situarla en un lugar prioritario como factor esencial y primordial en la etiología del uso de drogas. Es necesario desmontar el "mito" de que diversión y uso de drogas forman un binomio indisociable, en el cual el uso de drogas tiende a ser justificado en ocasiones a través de un concepto de diversión asociado a la búsqueda de sensaciones asociada al riesgo, la desinhibición y oposición ante toda norma convencional. La prevención y los estudios que constituyen su base podrían aprender mucho de la gestión del fin de semana que hacen los jóvenes y adolescentes no consumidores, y las campañas educativas podrían también volver su enfoque hacia estas experiencias que demuestran que una alta implicación y disfrute del fin de semana sin drogas es posible y está muy presente en nuestras comunidades. Es también necesario favorecer el desarrollo de entonos físicos en respuesta a las necesidades de muchos adolescentes y jóvenes que demandan entornos donde la diversión pueda conseguir sus objetivos esenciales y genuinos (socialización, estimulación, evasión y fiesta) sin mezclarse con el uso de drogas. Sin la generalización de estas experiencias será más difícil el logro de esta diversión genuina y que es una justa aspiración de muchos jóvenes.

En segundo lugar, es necesario tener en cuenta que el uso de drogas se inicia y mantiene a través de modelos e interacciones con el grupo de pares. El uso de drogas se justifica en ocasiones a través del vínculo afectivo (con familiares, amigos y relaciones íntimas, en una suerte de "parasitismo conceptual" similar al que ocurre con el concepto de diversión) e incluso se convierte en un criterio para la inclusión y exclusión en la red social del sujeto. La prevención del uso de drogas necesita desarrollar mensajes que ayuden a los jóvenes a discernir entre lo relevante de una relación afectiva y el uso de drogas como un componente accesorio y circunstancial. Una parte de la asociación entre el uso de drogas y estas dos áreas vitales básicas (la diversión y los vínculos afectivos con personas significativas) se explica por un estilo de vida orientado hacia la no-conformidad con las normas sociales, la conducta problema y los riesgos en general y el alejamiento de los valores convencionales. El soporte del uso de drogas se basa también en sesgos en la percepción y valoración en la utilidad del consumo de drogas, la motivación para consumirlas y las consecuencias asociadas. Muchos adolescentes y jóvenes presentan déficit cognitivos que impiden valorar los riesgos asociados al uso de drogas, (especialmente a medio y largo plazo) y éstos déficit (junto con características personales orientadas en contra de los valores convencionales) llevan en ocasiones a rechazar el control y regulación social del consumo. Muchos jóvenes consumidores de drogas distorsionan los motivos que tienen otros jóvenes para no consumir drogas, y atribuyen esta conducta a causas "defensivas" que son menos relevantes que otras causas relacionadas con un estilo de vida orientado hacia una concepción de uno mismo ("self") y de la comunidad donde el uso de drogas no tiene sentido, utilidad ni cabida. Algunos adolescentes y jóvenes presentan sesgos cognitivos consistentes en atribuir efectos al uso de drogas que llegan desde lo instrumental (especialmente 
en el espacio recreativo y relaciones interpersonales) hasta lo casi mágico (mejorar el bienestar personal y la experiencia vital, etc).

La modificación de estos esquemas y sesgos cognitivos que representan una vulnerabilidad ante el uso de drogas y sus riesgos asociados y el fomento de una diversión genuina e incontaminada con el uso de drogas pueden prevenir el uso de drogas en muchos jóvenes europeos. En otros casos, la prevención requerirá además modificar estilos de vida donde el uso de drogas forma parte de una orientación hacia la conducta problema, los riesgos y la falta de autocontrol más generalizados.

\section{REFERENCIAS}

Aitken, C.; Kerger, M.; Crofts, N.; (2000). Drivers Who Use Illicit Drugs: behaviour and perceived risks. Drugs: education, prevention and policy, $N^{0} 1,7,39-50$.

Becoña, E.; (1999). Bases teóricas que sustentan los programas de prevención de drogas. Madrid. Delegación del Gobierno para el Plan Nacional sobre Drogas.

Calafat, A., Stocco, P., Mendes, F., Simon, J., Van de Vijngaart, G., Sureda, P., Palmer, A., Maalsté, N., y Zavatti, P., (1998). Characteristics and social representation of ecstasy in Europe. Palma de Mallorca: Irefrea.

Calafat, A., Bohrn, K., Juan, M., Kokkevi, A., Maalsté, N., Mendes, F., Palmer, A., Sherlock, K., Simon, J., Stocco, P., Sureda, P., Tossman, P., Van de Vijngaart, G., y Zavatti, P. (2001). Night life in Europe and re creative drug use. SONAR 98. Palma de Mallorca: Irefrea.

Calafat, A., Juan, M., Becoña, E., Fernández, C., Gil, E., Palmer, A., Sureda, P. y Torres, M.A., (2000). Salir de marcha y consumo de drogas. Madrid: Plan Nacional sobre Drogas.

Calafat, A., Fernández, C., Juan, M., Bellis, M.A., Bohrn, K., Hakkarainen, P., Kilfoyle-Carrington, M., Kokkevi, A., Maalsté, N., Mendes, F., Siamou, I., Simon, J., Stocco, P. y Zavatti, P. (2001). Risk and control in the recreational drug culture. SONAR PROJECT. Palma de Mallorca: Irefrea.
Cummings, K.M.; Becker, M.H.; maile, M.; (1980). Bringing the models together: an empirical approach to combining variables used to explain health actions. Journal of Behavior Medicine, 3, 123-145.

Petraitis, J.; Flay, B.R.; Miller, T.Q.; (1995). Reviewing theories of adolescent substance use: Organizing pieces in the puzzle. Psychological Bulletin, 117, 67-86.

Hawkins, J.D.; Catalano, R.F.; Miller, J.L.; (1992). Risk and protective factors for alcohol and other drug problems in adolescence and early adulthood: Implications for substance abuse prevention. Psychological Bulletin, 112, 64105.

Jessor, R.; Van Den Bos, J.; Vanderryn, J.; Costa, F.M.; Turbin, M.S.; (1995). Protective factors in adolescent problem behavior: Moderator effects and developmental change. Developmental Psychology, 31, 923-933.

Leigh, B.C.; (1999). Peril, chance, adventure: concepts of risk, alcohol use and risky behavior in young adults. Addiction, 94 (3), 371-383.

Peele, S.; (1987). A Moral Vision of Addictions: how people's values determine whether they became and remain addicts. Drug Issues, 17(2), 187-215.

Pettet, J.R.; (1993). A Closer Look at the Role of a Spiritual Approach in Addictions Treatment. Journal of Substance Abuse Treatment, vol. 10, 263-267.

Rhodes, T.; Lilly, R.; Fernández, C.; Giorgino, E.; Kemmesis, U.E.; Ossebaard, H.C.; Lalam, N.; Faasen, I.; Spannow, K.E.; Wiessing, L.; (1999). Risk factors associated with drug use: the importance of "risk environment". European Monitoring Centre for Drugs and Drug Addiction. Available at www.emcdda.org/activities/ epidem_analyse_risk.shtml

Rogers, R.W.; (1984). Cognitive and physiological processes in fear appeals and attitude change. A revised theory of protection motivation. En: Cacioppo, J.T.; Petty, R.E. (Eds.): Social Psychophysiology, 153-176. new York: Guilford Press.

Rokeach, M.A.; (1979). Human Values, Smoking Behavior, and Public Health Programs. En M. Rokeach: Understanding Human Values. Individual and Societal. The Free Press. pp. 199209.

Weinstein, N.D.; (1993). Testing four competing theories of health-protective behavior. Health Psychology, 12, 324-333. 
